\title{
Research Article \\ Covariant Canonical Method for Yang-Mills Theory Expressed as a Constrained BF-Like Theory
}

\author{
Alberto Escalante and Irving García \\ Instituto de Física Luis Rivera Terrazas, Benemérita Universidad Autónoma de Puebla (IFUAP), \\ Apartado Postal J-48, 72570 Puebla, PUE, Mexico \\ Correspondence should be addressed to Alberto Escalante, aescalan@ifuap.buap.mx
}

Received 2 March 2012; Accepted 18 May 2012

Academic Editor: Shao-Ming Fei

Copyright (C) 2012 A. Escalante and I. García. This is an open access article distributed under the Creative Commons Attribution License, which permits unrestricted use, distribution, and reproduction in any medium, provided the original work is properly cited.

\begin{abstract}
The covariant canonical analysis for Yang-Mills theory expressed as a BF-like action is performed. We study a BF-like action, that in spite of being the coupling of two topological terms, yield, on shell to Yang-Mills action. In addition, by using the results obtained in the covariant canonical approach we study the symmetries of the action, in particular we calculate its energy-momentum tensor obtaining the same tensor found for Yang-Mills theory; then we confirm those results by using Noether's theorem.
\end{abstract}

\section{Introduction}

For studying a dynamical system, it is mandatory to know its symmetries at classical and quantum level in order to research the relevant physical implications of them. Nowadays, there exist approaches that can be used for studying extensively the symmetries of a given theory as for instance, Dirac's canonical formalism and the covariant canonical method, both with its respective advantages. Dirac's canonical formalism is an elegant approach for obtaining relevant physical information of a theory under study namely, the counting of physical degrees of freedom, the gauge transformations, the study of the constraints, the obtention of the extended Hamiltonian and the extended action [1, 2], being a relevant information it is the guideline to make the best progress in the analysis of quantum aspects. On the other hand, in the covariant canonical method in order to describe all the relevant Hamiltonian description of the covariant phase space [3-5], we are able to identify a closed and gauge invariant two-form, being an important step to analyze within a complete covariant context the theory under study. However, it is well known that the analysis of a dynamical system within the covariant canonical formalism is based on the statement that 
the classical phase space of a given physical theory is defined as the space of solutions of its classical equations of motion, but this implies that the phase space is not endowed with a natural or preferred symplectic structure as has been claimed in [6], and the freedom in the choice of the symplectic structure is an important fact because could yield to different quantum mechanics formulations [6-8]. Nonetheless, this inconvenience can be overcome if we extend the definition of a dynamical system by considering its equations of motion plus a principle action, thus we are in a profitable situation because the action gives us the equations of motion; from the other side it fixes the symplectic structure of the theory $[8,9]$. Thus, in the study of the symmetries of a dynamical system must be taken into account both, the equations of motion plus an action principle. Therefore, we think that the complete analysis of a dynamical system should be done by performing the Dirac's canonical approach and the canonical covariant method by taking into account the action principle and its equations of motion. In order to emphasize these points, let us consider the following action which is the subject of this paper:

$$
S_{1}[B, A]=\int_{M}\left[\frac{1}{4} B^{I}{ }_{\mu \nu} B_{I}{ }^{\mu \nu}-\frac{1}{2} B_{I}{ }^{\mu \nu}\left(\partial_{\mu} A_{\nu}^{I}-\partial_{\nu} A_{\mu}^{I}+f_{J K}^{I} A_{\mu}^{J} A_{\nu}^{K}\right)\right] d x^{4},
$$

where $B^{I}$ is a set of $\left(N^{2}-1\right)$ valued $\mathrm{SU}(N)$ 2-forms and $F^{I}$ is the curvature of the connection 1-form $A^{I}$ (see below). This action has been studied in the context of Dirac's analysis in [10], concluding that the action has the same number of physical degrees of freedom than standard Yang-Mills action [YM]; however, it is clear that standard [YM] action and (1.1) presents a different structure, so, we are in the situation that two different actions yield the same equations of motion. Nevertheless, we have commented above that the equations of motion are not an enough element to develop a complete study of the symmetries of a dynamical system. In fact, standard [YM] action and (1.1) describe, on shell, the same classical description, but the next question rise: what about the symplectic geometry?

The aim of the present paper is focused on the context of the covariant canonical formalism, we develop the covariant canonical study of the action (1.1) taking into account the equations of motion and the action principle. We obtain along the paper a closed and gauge invariant geometric structure which in turns represents a complete Hamiltonian description of the covariant phase space, and we show that the action (1.1) in fact, corresponds at classical level to [YM] theory. In addition, by using the former results we calculate for (1.1) the corresponding energy-momentum tensor, reproducing on shell the symmetric and gauge invariant energy-momentum tensor reported for [YM] theory. It is important to remark, that in order to calculate the energy-momentum tensor, usually it is calculated by using the standard canonical tensor [11] namely, $T_{\mu}{ }^{v}=\left(\partial L / \partial \eta_{\rho, v}\right) \eta_{\rho \mu}-\delta_{\mu}^{v} L$, where $L$ represents the Lagrangian density, and $\eta_{\rho}$ are field variables. However, it is well known that the energy-momentum tensor obtained in the canonical approach, in general is neither symmetric nor gauge invariant, and usually Belinfante's method is required to symmetrize the tensor. Nonetheless, in order to obtain a symmetric and gauge invariant tensor without resort to Belinfante's method, we must apply correctly Noether's theorem, this means, we must take into account the action principle and the full equations of motion of the system under study [12]. In this respect, as a complementary part of this paper, we calculate the energy-momentum tensor for the action (1.1) by using Noether's theorem, and we reproduce on shell the symmetric and gauge invariant energy-momentum tensor for [YM] 
theory; thus, we confirm by means of a different way the results obtained in the covariant canonical approach.

\section{Covariant Canonical Method for [YM] Theory Expressed as a BF-Like Theory}

The action that we shall study in this section is given by [10]

$$
S[A, B]=\int_{M} * B_{I} \wedge B^{I}-2 B_{I} \wedge * F^{I},
$$

where $B^{I}=(1 / 2) B^{I}{ }_{\mu \nu} d x^{\mu} \wedge d x^{\nu}$ is a set of $\left(N^{2}-1\right) \mathrm{SU}(N)$ valued two-forms, $F^{I}=$ $(1 / 2) F_{\mu \nu}^{I} d x^{\mu} \wedge d x^{\nu}$, with $F_{\mu \nu}^{I}=\partial_{\mu} A_{\nu}^{I}-\partial_{\nu} A_{\mu}^{I}+f^{I}{ }_{J K} A_{\mu}^{J} A_{\nu}^{K}$ is the curvature of the connection 1 -form $A^{I}=A_{\mu}^{I} d x^{\mu}$. Here, $\mu, v=0,1, . ., 3$ are spacetime indices, $x^{\mu}$ are the coordinates that label the points for the 4-dimensional Minkowski manifold $M$, and $*$ is the spacetime Hodgeduality operation.

The action takes the following form:

$$
S[B, A]=\int_{M}\left[\frac{1}{4} B^{I}{ }_{\mu \nu} B_{I}^{\mu \nu}-\frac{1}{2} B_{I}^{\mu \nu}\left(\partial_{\mu} A_{v}^{I}-\partial_{\nu} A_{\mu}^{I}+f_{J K}^{I} A_{\mu}^{J} A_{v}^{K}\right)\right] d x^{4}
$$

In order to perform the covariant canonical analysis, we start calculating the variation of the action (2.2), obtaining

$$
\delta S[B, A]=\int_{M}\left[\frac{1}{2}\left(B^{I}{ }_{\mu \nu}-F^{I}{ }_{\mu \nu}\right) \delta B_{I}{ }^{\mu \nu}+D_{\mu} B_{I}{ }^{\mu v} \delta A_{v}^{I}-\partial_{\mu}\left(B_{I}{ }^{\mu v} \delta A_{v}^{I}\right)\right] d x^{4},
$$

where the covariant derivative is defined by $D_{\alpha} \lambda_{\mu}^{I}=\partial_{\alpha} \lambda_{\mu}^{I}+f^{I}{ }_{J K} A_{\alpha}^{J} \lambda_{\mu}^{K}$. From (2.3), we identify the following equations of motion:

$$
\begin{gathered}
B_{\mu \nu}^{I}=F_{\mu \nu \prime}^{I} \\
D_{\mu} B^{\mu \nu I}=0,
\end{gathered}
$$

and the pure divergence term of the variation:

$$
\Psi^{\mu}=B_{I}^{\mu v} \delta A_{v}^{I}
$$

corresponds to the symplectic potential for the theory which does not contribute locally to the dynamics, but generates the symplectic form on the covariant phase space [5]. We observe that by substituting the first equation of motion in the second one, (2.4) is reduced on shell to the usual [YM] equations of motion, we can appreciate at this level the double role of the action (2.2), as we have already commented above, it gives us the equations of motion (2.4), and also it fixes the symplectic geometry through (2.5). Furthermore, we would comment 
that the action (2.2) has the important characteristic of being the coupling of two topological theories in the sense that these terms are lacking if degrees of freedom. There is another definition for a topological theory as that independent of the metric; however, in this work we call topological that being devoid of local degrees of freedom. That is, the theory is susceptible only to global degrees of freedom associated with nontrivial topologies of the manifold in which they are defined and topologies of the gauge bundle [7]. In fact, the first term of (2.1) $\left(* B_{I} \wedge B^{I}\right)$ is devoid of physical degrees of freedom, and the second one $\left(B_{I} \wedge * F^{I}\right)$ as well [10]. In this manner, the coupling of two topological theories defines a dynamical theory in the sense that in (2.1) there exist physical degrees of freedom; those of [YM] theory [10], this feature of (2.1) is present also in Plebanski theory of gravity, where Plebanski action is the coupling of two topological terms as well $[13,14]$.

For our aims, we define the fundamental concept in the studio of the canonical covariant formalism: the covariant phase space for the theory described by (2.1) is the space of solutions of (2.4), and it will be called Z. Furthermore, we need to obtain a closed and gauge invariant geometric structure on $Z$.

To this aim, we are able to obtain the integral kernel of the geometric structure for the theory by means of the variation (the functional exterior derivative on $Z$, see [7]) of the symplectic potential (2.5), obtaining

$$
\omega=\int_{\Sigma} J^{\mu} d \Sigma_{\mu}=\int_{\Sigma} \delta \Psi^{\mu} d \Sigma_{\mu}=\int_{\Sigma} \delta B_{I}^{\mu v} \wedge \delta A_{\nu}^{I} d \Sigma_{\mu}
$$

where $\Sigma$ is a Cauchy surface.

On the other side, we need to prove that our symplectic form on $Z$ is a (nondegenerate) closed two-form; the closeness is equivalent to the Jacobi identity in the conventional Hamiltonian scheme, and the antisymmetry of a two-form represents the antisymmetry of Poisson brackets. Moreover, the integral kernel of the geometric form $J^{\mu}$ is conserved $\left(D_{\mu} J^{\mu}=0\right)$, which guarantees that $\omega$ is independent of $\Sigma$. In fact, we can observe that $B_{I}{ }^{\mu \nu}$ and $A_{v}^{I}$ correspond to 0 -forms on $Z$, and $\delta B_{I}^{\mu \nu}, \delta A_{v}^{I}$ are 1 -forms on $Z$, thus our symplectic structure is a two-form. On the other side, to prove the closeness of $\omega$, we see that $\delta^{2} A_{v}^{I}=0$ and $\delta^{2} B_{I}{ }^{\mu \nu}=0$ because $A_{v}^{I}$ and $B_{I}{ }^{\mu \nu}$ are independent 0 -forms on the covariant phase space $Z$ and $\delta$ is nilpotent, so by using this fact in $\omega$ we find that

$$
\delta \omega=\int_{\Sigma}\left[\delta^{2} B_{I}^{\mu \nu} \wedge \delta A_{v}^{I}-\delta B_{I}^{\mu \nu} \wedge \delta^{2} A_{v}^{I}\right] d \Sigma_{\mu}=0
$$

In order to prove that $J^{\mu}$ defined in (2.6) is conserved, it is necessary to calculate the linearized equations of motion. For this aim, we replace in (2.4) $A_{\mu}^{I} \rightarrow \delta A_{\mu}^{I}$ and $B^{I}{ }_{\mu \nu} \rightarrow \delta B^{I}{ }_{\mu \nu}$, keeping to first order in $\delta$, the linearized equations are given by

$$
\begin{gathered}
\delta B^{I}{ }_{\mu \nu}=D_{\mu} \delta A_{v}^{I}-D_{\nu} \delta A_{\mu \prime}^{I} \\
D_{\mu} \delta B_{I}^{\mu \nu}+f_{I J}{ }^{K} \delta A_{\mu}^{J} B_{K}^{\mu v}=0 .
\end{gathered}
$$


We also need observe that under the gauge transformations of the theory $A_{\mu}^{I} \rightarrow A_{\mu}^{I}-D_{\mu} \epsilon^{I}$ and $B_{\mu \nu}^{I} \rightarrow B_{\mu \nu}^{I}+f_{L J}^{I} B^{J}{ }_{\mu \nu} \epsilon^{L}$ [10], for some infinitesimal variation we have

$$
\begin{gathered}
\delta A^{\prime I}=\delta A_{\mu}^{I}-f_{J K}^{I} \delta A_{\mu}^{J} \epsilon^{K}, \\
\delta B_{I}^{\prime \mu \nu}=\delta B_{I}{ }^{\mu \nu}+f_{I L}{ }^{K} \delta B_{K}{ }^{\mu v} \epsilon^{L} .
\end{gathered}
$$

In this manner, by using (2.9) one obtains that $\omega$ transforms

$$
\omega^{\prime}=\int_{\Sigma} \delta B_{I}^{\prime \mu \nu} \wedge \delta A_{\nu}^{\prime I} d \Sigma_{\mu}=\omega+\int O\left(\epsilon^{2}\right) d \Sigma
$$

Therefore, $\omega$ is an $\mathrm{SU}(N)$ singlet. This result allows us to prove that

$$
\begin{aligned}
\partial_{\mu} J^{\mu} & =D_{\mu} J^{\mu}=D_{\mu}\left(\delta B_{I}{ }^{\mu \nu}\right) \wedge \delta A_{v}^{I}+\delta B_{I}{ }^{\mu \nu} \wedge \delta D_{\mu} A_{v}^{I} \\
& =-f_{I J K} B^{K \mu \nu} \delta A_{\mu}^{J} \wedge \delta A_{v}^{I}+\frac{1}{2} \delta B_{I}{ }^{\mu \nu} \wedge \delta B^{I}{ }_{\mu \nu}=0
\end{aligned}
$$

where we have used the linearized equations (2.8) and the antisymmetry of 1-forms $\delta A_{v}^{I}$ and $\delta B_{I}{ }^{\mu \nu}$ on the phase space.

Thus, with the above results if we perform a Lorentz transformation $\Sigma_{t} \rightarrow \Sigma_{t}^{\prime}$ and $\omega \rightarrow \omega^{\prime}$, that is,

$$
\omega^{\prime}=\int_{\Sigma_{t}^{\prime}} J^{\prime \mu} d \Sigma_{\mu}^{\prime}=\int_{\Sigma_{t}} J^{\mu} d \Sigma_{\mu}=\omega
$$

It follows that $\omega$ given in (2.6) is Lorentz invariant. In this manner, with these results we have constructed a Lorentz and gauge invariant symplectic structure on the phase space, and it is possible to formulate the Hamiltonian theory in a manifestly covariant way.

Now, by considering that upon picking $\Sigma$ to be the standard initial value surface at $t=0$, (2.6) takes the form

$$
\omega=\int_{\Sigma} \delta \Pi_{I}^{a} \wedge \delta A_{a}^{I}
$$

where $\Pi_{I}^{a} \equiv B_{I}^{0 a}$ and $a, b, c=1,2,3$.

For two 0 -forms $f, g$ defined on $Z$, the Hamiltonian vector field defined by the symplectic structure (2.13) is given by [15]

$$
X_{f}=\int_{\Sigma} \frac{\delta f}{\delta \Pi_{I}^{a}} \frac{\delta}{\delta A^{I} a}-\frac{\delta f}{\delta A_{a}^{I}} \frac{\delta}{\delta \Pi_{I}^{a}}
$$


and the Poisson bracket $\{f, g\}:=-X_{f}(g)$ is given by

$$
\{f, g\}=\int_{\Sigma} \frac{\delta f}{\delta A^{I}{ }_{a}} \frac{\delta g}{\delta \Pi_{I}{ }^{a}}-\frac{\delta f}{\delta \Pi_{I}{ }^{a}} \frac{\delta g}{\delta A^{I}{ }_{a}}
$$

In this way, smearing the constraints of this theory with test fields [10]:

$$
\gamma_{I}\left[P^{I}\right]:=\int_{\Sigma} P^{I}\left(D_{a} \Pi_{I}^{a}\right)
$$

By inspection, the functional derivatives different to zero are given by

$$
\frac{\delta \gamma_{I}\left[P^{I}\right]}{\delta \Pi_{I}^{a}}=-D_{a} P^{I}, \quad \frac{\delta \gamma_{I}\left[P^{I}\right]}{\delta A_{a}^{I}}=P^{L} f_{L I} K^{K} \Pi_{K}^{a}
$$

Thus, using (2.13) and (2.14) we observe that the motion on $Z$ generated by $\gamma_{I}\left[P^{I}\right]$ is given by

$$
\begin{gathered}
A_{a}^{I} \longrightarrow A_{a}^{I}-\epsilon D_{a} P^{I}+O\left(\epsilon^{2}\right), \\
\Pi_{I}^{a} \longrightarrow \Pi_{I}^{a}-\epsilon P^{L} f_{L I}{ }^{K} \Pi_{K}^{a}+O\left(\epsilon^{2}\right),
\end{gathered}
$$

where $\epsilon$ is an infinitesimal parameter [15]. We can see that the later gauge transformation (2.18) corresponds to those found by using Dirac's canonical method reported in [10].

Therefore, as a conclusion of this part, we have constructed a closed and gauge invariant symplectic form on $Z$ which in turns represent a complete Hamiltonian description of the covariant phase space for the theory. These results will allow us to analyze the quantum treatment in forthcoming works by means of an alternative way with respect to that reported in [10].

Now, the next question a rises: is the energy-momentum tensor for the theory (2.1) the same as found for [YM] theory? We have seen that the action (2.1) share the same equations of motion with the usual action of [YM]; however, this fact does not imply that the energymomentum tensor will be the same. In fact, we are able to find in the literature that two actions yielding to the same equations of motion, their corresponding energy-momentum tensors are quite different [7]. With the results presented above we can answer that question. For this aim, we remember that the symplectic structure have emerged from the variation of the symplectic potential, that is,

$$
\psi^{\mu}=B_{I}^{\mu \nu} \delta A_{v}{ }^{I}
$$

thus, by making the contraction of $\omega$ with the vector field $V=\epsilon^{\mu} \partial_{\mu}$, where $\epsilon^{\mu}$ corresponds to a constant spacetime vector, and using the following identity:

$$
V\rfloor \omega=\left\llcorner_{v} \psi-\delta(v\rfloor \psi\right),
$$


we find

$$
\begin{aligned}
\mathcal{L}_{V} \psi^{\mu} & =\mathcal{L}_{V} B_{I}{ }^{\mu \nu} \delta A_{v}{ }^{I}+B_{I}{ }^{\mu v} \mathcal{L}_{V} \delta A_{v}{ }^{I} \\
& \left.=\epsilon^{\alpha} \partial_{\alpha} B_{I}{ }^{\mu \nu} \delta A_{v}{ }^{I}+B_{I}{ }^{\mu v} \epsilon^{\alpha} \partial_{\alpha} \delta A_{v}{ }^{I}, V\right] \psi^{\mu}=B_{I}{ }^{\mu v} \epsilon^{\alpha} F^{I}{ }_{\alpha \nu} .
\end{aligned}
$$

Therefore, the contraction of $V$ with the geometric structure gives

$$
\begin{aligned}
V] \omega= & \epsilon^{\alpha} \partial_{\alpha} B_{I}{ }^{\mu v} \delta A_{v}{ }^{I}+B_{I}{ }^{\mu v} \epsilon^{\alpha} \partial_{\alpha} \delta A_{v}{ }^{I}-\delta\left(B_{I}{ }^{\mu v} \epsilon^{\alpha} F^{I}{ }_{\alpha \nu}\right) \\
= & \partial_{\alpha}\left(\epsilon^{\alpha} B_{I}{ }^{\mu v} \delta A_{v}{ }^{I}-\epsilon^{\mu} B_{I}{ }^{\alpha v} \delta A_{v}{ }^{I}\right)+\epsilon^{\mu} D_{\alpha} B_{I}{ }^{\alpha v} \delta A_{v}{ }^{I} \\
& +\frac{1}{2} \epsilon^{\mu} B_{I}{ }^{\alpha \nu}\left(D_{\alpha} \delta A_{v}^{I}-D_{v} \delta A_{\alpha}^{I}\right)-\delta\left(B_{I^{\mu v}} \epsilon^{\alpha} F^{I}{ }_{\alpha v}\right) .
\end{aligned}
$$

So, from the equation of motion (2.4), we obtain the desired result:

$$
\begin{aligned}
V] \omega & =\frac{1}{4} \epsilon^{\mu} \delta\left(F_{I}^{\alpha v} F_{\alpha \nu}^{I}\right)-\delta\left(\epsilon^{\alpha} F_{I}{ }^{\mu v} F^{I}{ }_{\alpha \nu}\right) \\
& =-\delta\left[\epsilon^{\alpha}\left(F_{I}^{\mu v} F^{I}{ }_{\alpha \nu}-\frac{1}{4} \eta^{\mu}{ }_{\alpha} F_{I}{ }^{\alpha v} F_{\alpha \nu}^{I}\right)\right],
\end{aligned}
$$

where we are able to identify the energy-momemtum tensor for the the action (2.1) given by

$$
T_{\alpha}^{\mu}=F_{I}^{\mu \nu} F_{\alpha \nu}^{I}-\frac{1}{4} \eta_{\alpha}^{\mu} F_{I}^{\alpha \nu} F_{\alpha \nu}^{I}
$$

that correspond to the energy-momentum tensor for [YM] theory [11]. Therefore, the action (2.1) written as a BF-like theory reproduces on shell the [YM] energy-momentum tensor. It is important to remark that this result is no longer true off shell, and this fact could yield to the presence of monopoles charges in the vacuum as is present in other BF-like models for [YM] [16]. On the other hand, we can appreciate that by using the covariant canonical approach, the obtained energy-momentum tensor is symmetric and gauge invariant, and we have not used alternative suppositions as Belinfante's approach in order to symmetrize it. As a complement part of this work, in latter sections we will obtain the same energy-momentum tensor by using Noether's theorem. Furthermore, it is important to remark that the action studied in this part could be useful to research the coupling of matter fields with a pure BF theory; however, this subject is still in progress.

\section{Energy-Momentum Tensor for [YM] Theory Expressed as a BF-Like Theory by Using Noether's Theorem}

In this section, we will use Noether's theorem in order to find the energy-momentum tensor for the theory under study, we will confirm the results previously discussed. On the other hand, it is a nice exercise for applying such theorem to theories with more dynamical variables than conventional [YM] theory [12]. 
Let us start with the following action:

$$
S\left[A_{\mu}^{I}, B_{I \mu \nu}\right]=\int_{R}\left[\frac{1}{4} \alpha B_{\mu \nu}^{I} B_{I}^{\mu \nu}-\frac{1}{2} \alpha B_{I}^{\mu \nu} F_{\mu \nu}^{I}\right] d x^{4},
$$

where the following Lagrangian density can be identified:

$$
\mathcal{L}=\frac{1}{4} \alpha B_{\mu \nu}^{I} B_{I}^{\mu \nu}-\frac{1}{2} \alpha B_{I}^{\mu \nu} F_{\mu \nu}^{I}
$$

here, $R$ is an arbitrary region of the four-dimensional Minkowski spacetime $M$. We can observe that the dynamical variables for the theory are $\left(A_{\mu}^{I}, B_{\mu \nu}^{I}\right)$ and as dynamical ones, both have a contribution to the energy-momentum tensor, in other words, they should be treated on an equal footing. So, if we take the variation of the action (3.1) under the transformation of the variables $\widetilde{\delta} A_{\mu}^{I}:=\widetilde{A}_{\mu}^{I}-A_{\mu}^{I}$ and $\widetilde{\delta} B_{I \mu \nu}:=\widetilde{B}_{I \mu \nu}-B_{I \mu \nu}[11,12]$, then we obtain the following result:

$$
\tilde{\delta} S=\int_{R} d^{4} x\left[\frac{\partial \mathcal{L}}{\partial B_{I \mu \nu}} \tilde{\delta} B_{I \mu v}-\partial_{v}\left(\frac{\partial \mathcal{L}}{\partial\left(\partial_{\nu} A_{\mu}^{I}\right)}\right) \tilde{\delta} A_{\mu}^{I}\right]+\int_{\partial M^{4}} d \Sigma_{v}\left(\frac{\partial \mathcal{L}}{\partial\left(\partial_{v} A_{\mu}^{I}\right)} \tilde{\delta} A_{\mu}^{I}\right)
$$

Thus, $\widetilde{\delta} S=0$ yields the equations of motion:

$$
\begin{gathered}
\frac{\delta S}{\delta B_{I \mu \nu}} \equiv \frac{\partial \mathcal{L}}{\partial B_{I \mu \nu}}=\frac{1}{2} \alpha B^{I \mu \nu}-\frac{1}{2} \alpha F^{I \mu \nu}=0 \\
\frac{\delta S}{\delta A_{\mu}^{I}} \equiv-D_{\nu}\left(\frac{\partial \mathcal{L}}{\partial\left(\partial_{\nu} A_{\mu}^{I}\right)}\right)=\alpha D_{\nu} B_{I}^{\mu \nu}=0 .
\end{gathered}
$$

We see that

$$
\begin{aligned}
D_{v}\left(\frac{\delta S}{\delta B_{I \mu \nu}}\right) & =\frac{1}{2} \alpha D_{v} B^{I \mu \nu}-\frac{1}{2} \alpha D_{v} F^{I \mu \nu} \\
& =\frac{1}{2} \frac{\delta S}{\delta A_{\mu}^{I}}-\frac{1}{2} \alpha D_{v} F^{I \mu \nu},
\end{aligned}
$$

this expression will be used in later calculations.

The action (3.1) is invariant under the Poincaré group, and Noether's theorem demands the infinitesimal version of the Poincaré transformations are given by

$$
x^{\prime \mu}=x^{\mu}+\delta x^{\mu}, \quad \delta x^{\mu}=\varpi^{\mu}{ }_{\nu} x^{\nu}+\epsilon^{\mu},
$$


where $\varpi_{v}^{\mu}{ }_{v}=-\varpi_{v}{ }^{\mu}$ and $\epsilon^{\mu}$ are the infinitesimal arbitrary constant parameters [11]. Therefore, to first order we have the following transformations

$$
\begin{aligned}
& F_{I \mu \nu}^{\prime}\left(x^{\prime}\right)=F_{I \mu \nu}+\left(\partial_{\nu} \delta x^{\beta}\right) F_{I \beta \mu}+\left(\partial_{\mu} \delta x^{\beta}\right) F_{I \nu \beta,} \\
& B_{I \mu v}^{\prime}\left(x^{\prime}\right)=B_{I \mu v}+\left(\partial_{\nu} \delta x^{\beta}\right) B_{I \beta \mu}+\left(\partial_{\mu} \delta x^{\beta}\right) B_{I \nu \beta}, \\
& B_{I \mu \nu}^{\prime} B^{\prime I \mu \nu}=B_{I \mu \nu} B^{I \mu \nu}+4\left(\partial_{\nu} \delta x^{\beta}\right) B_{I \beta \mu} B^{I \mu \nu}, \\
& B_{I \mu \nu}^{\prime}{F^{I \mu \nu}}^{I \mu}=B_{I \mu \nu} F^{I \mu \nu}+2 B^{I \mu \nu}\left(\partial_{\nu} \delta x^{\beta}\right) F_{I \beta \mu}+2\left(\partial_{\nu} \delta x^{\beta}\right) B_{I \beta \mu} F^{I \mu \nu}
\end{aligned}
$$

thus, the transformed action is given by

$$
\begin{aligned}
S^{\prime}= & \int_{\mathcal{R}^{\prime}} d^{4} x^{\prime} \mathcal{L}^{\prime}=\int_{\mathcal{R}} d^{4} x\left[\mathcal{L}+\alpha\left(\partial_{\nu} \delta x^{\beta}\right) B_{I \beta \mu} B^{I \mu \nu}-\alpha B^{I \mu \nu}\left(\partial_{\nu} \delta x^{\beta}\right) F_{I \beta \mu}-\alpha\left(\partial_{\nu} \delta x^{\beta}\right) B_{I \beta \mu} F^{I \mu \nu}\right] \\
= & \int_{\mathcal{R}} \mathcal{L} d^{4} x+\int_{\mathcal{R}} \mathcal{L} d^{4} x\left(\partial_{\beta} \delta x^{\beta}\right) \\
& +\int_{R} d^{4} x\left[\alpha\left(\partial_{\nu} \delta x^{\beta}\right) B_{I \beta \mu} B^{I \mu \nu}-\alpha B^{I \mu \nu}\left(\partial_{\nu} \delta x^{\beta}\right) F_{I \beta \mu}-\alpha\left(\partial_{\nu} \delta x^{\beta}\right) B_{I \beta \mu} F^{I \mu \nu}\right] \\
= & +\int_{R} \mathcal{L} d^{4} x\left(\partial_{\beta} \delta x^{\beta}\right)+\int_{R} d^{4} x \partial_{v}\left[\alpha \delta x^{\beta}\left(B_{I \beta \mu} B^{I \mu \nu}-B^{I \mu \nu} F_{I \beta \mu}-B_{I \beta \mu} F^{I \mu \nu}\right)\right] \\
& -\int_{R} d^{4} x \alpha \delta x^{\beta} \partial_{\nu}\left(B_{I \beta \mu} B^{I \mu \nu}-B^{I \mu \nu} F_{I \beta \mu}-B_{I \beta \mu} F^{I \mu \nu}\right) .
\end{aligned}
$$

For our aims, we need to rewrite the last three terms of (3.8) as follows.

The term:

$$
\begin{aligned}
-\alpha \delta x^{\beta} \partial_{v}\left(B_{\beta \mu}^{I} B_{I}^{\mu \nu}\right)= & -\alpha \delta x^{\beta}\left(\partial_{\nu} B_{\beta \mu}^{I}\right) B_{I}^{\mu \nu}-\alpha \delta x^{\beta} B_{\beta \mu}^{I} \partial_{\nu} B_{I}^{\mu \nu} \\
= & -\alpha \delta x^{\beta}\left(D_{v} B_{\beta \mu}^{I}\right) B_{I}^{\mu \nu}-\alpha \delta x^{\beta} B_{\beta \mu}^{I} D_{v} B_{I}^{\mu \nu} \\
= & -\frac{1}{2} \alpha \delta x^{\beta}\left(D_{v} B_{\beta \mu}^{I}-D_{\mu} B_{\beta \nu}^{I}\right) B_{I}^{\mu \nu}-\delta x^{\beta} B_{\beta \mu}^{I} \frac{\delta S}{\delta A_{\mu}^{I}} \\
= & -\frac{1}{2} \alpha \delta x^{\beta}\left(D_{v} B_{\beta \mu}^{I}+D_{\mu} B_{\nu \beta}^{I}+D_{\beta} B_{\mu \nu}^{I}\right) B_{I}^{\mu \nu} \\
& +\frac{1}{2} \alpha \delta x^{\beta} B_{I}^{\mu \nu} D_{\beta} B_{\mu \nu}^{I}-\delta x^{\beta} B_{\beta \mu}^{I} \frac{\delta S}{\delta A_{\mu}^{I}} \\
= & -\frac{1}{2} \alpha \delta x^{\beta}\left(\frac{1}{2} \varepsilon_{\rho \mu \nu \beta} \varepsilon^{\rho \sigma \gamma \lambda} D_{\sigma} B_{\gamma \lambda}^{I}\right) B_{I}^{\mu \nu} \\
& -\delta x^{\beta} B_{\beta \mu}^{I} \frac{\delta S}{\delta A_{\mu}^{I}}+\frac{1}{2} \alpha \delta x^{\beta} B_{I}^{\mu \nu} D_{\beta} B_{\mu \nu}^{I}
\end{aligned}
$$


and the last term of (3.9) can be written as

$$
\begin{aligned}
\frac{1}{2} \alpha \delta x^{\beta} B_{I}^{\mu v} D_{\beta} B_{\mu \nu}^{I} & =\frac{1}{4} \alpha \delta x^{\beta} D_{\beta}\left(B_{I}^{\mu v} B_{\mu \nu}^{I}\right) \\
& =\frac{1}{4} \partial_{\beta}\left(\alpha \delta x^{\beta} B_{I}^{\mu v} B_{\mu \nu}^{I}\right)-\frac{1}{4} \alpha B_{I}^{\mu v} B_{\mu \nu}^{I} \partial_{\beta} \delta x^{\beta}
\end{aligned}
$$

The term $\alpha \delta x^{\beta} \partial_{v}\left(B_{I}^{\mu \nu} F_{\beta \mu}\right)$ of (3.8):

$$
\begin{aligned}
& \alpha \delta x^{\beta} \partial_{v}\left(B_{I}^{\mu \nu} F_{\beta \mu}\right)=\alpha \delta x^{\beta} \partial_{v}\left(B_{I}^{\mu \nu}\right) F_{\beta \mu}^{I}+\alpha \delta x^{\beta} B_{I}^{\mu \nu} \partial_{v} F_{\beta \mu}^{I} \\
& =\alpha \delta x^{\beta} D_{v}\left(B_{I}^{\mu \nu}\right) F_{\beta \mu}^{I}+\alpha \delta x^{\beta} B_{I}^{\mu \nu} D_{\nu} F_{\beta \mu}^{I} \\
& =\delta x^{\beta} \frac{\delta S}{\delta A_{\mu}^{I}} F_{\beta \mu}^{I}+\frac{1}{2} \alpha \delta x^{\beta} B_{I}^{\mu \nu}\left(D_{\nu} F_{\beta \mu}^{I}+D_{\mu} F_{\nu \beta}^{I}+D_{\beta} F_{\mu \nu}^{I}\right)-\frac{1}{2} \alpha \delta x^{\beta} B_{I}^{\mu v} D_{\beta} F_{\mu \nu}^{I} \\
& =\delta x^{\beta} \frac{\delta S}{\delta A_{\mu}^{I}} F_{\beta \mu}^{I}+\frac{1}{2} \alpha \delta x^{\beta} B_{I}^{\mu v} \Psi_{\mu \nu \beta}^{I}-\frac{1}{2} \alpha \delta x^{\beta} B_{I}^{\mu v} D_{\beta} F_{\mu \nu}^{I},
\end{aligned}
$$

where $\Psi_{\mu \nu \beta}^{I}=D_{\mu} F_{\nu \beta}^{I}+D_{\nu} F_{\beta \mu}^{I}+D_{\beta} F_{\mu \nu}^{I}$ is the Bianchi's identity.

The last term of (3.8) can be written as

$$
\begin{aligned}
& \alpha \delta x^{\beta} \partial_{v}\left(B_{\beta \mu}^{I} F_{I}^{\mu \nu}\right)=\alpha \delta x^{\beta}\left(\partial_{v} B_{\beta \mu}^{I}\right) F_{I}^{\mu v}+\alpha \delta x^{\beta} B_{\beta \mu}^{I} \partial_{v} F_{I}^{\mu v} \\
& =\alpha \delta x^{\beta}\left(D_{v} B_{\beta \mu}^{I}\right) F_{I}^{\mu \nu}+\alpha \delta x^{\beta} B_{\beta \mu}^{I} D_{v} F_{I}^{\mu \nu} \\
& =\frac{1}{2} \alpha \delta x^{\beta}\left(D_{v} B_{\beta \mu}^{I}+D_{\mu} B_{\nu \beta}^{I}+D_{\beta} B_{\mu \nu}^{I}\right) F_{I}^{\mu \nu} \\
& -\frac{1}{2} \alpha \delta x^{\beta}\left(D_{\beta} B_{\mu \nu}^{I}\right) F_{I}^{\mu v}+\alpha \delta x^{\beta} B_{\beta \mu}^{I} D_{v} F_{I}^{\mu \nu} \\
& =\frac{1}{2} \alpha \delta x^{\beta}\left(\frac{1}{2} \varepsilon_{\rho \mu \nu \beta} \varepsilon^{\rho \sigma \gamma \lambda} D_{\sigma} B_{\gamma \lambda}^{I}\right) F_{I}^{\mu \nu}+\alpha \delta x^{\beta} B_{\beta \mu}^{I} D_{v} F_{I}^{\mu v} \\
& -\frac{1}{2} \alpha \delta x^{\beta}\left(D_{\beta} B_{\mu \nu}^{I}\right) F_{I}^{\mu \nu} \\
& =\frac{1}{2} \delta x^{\beta}\left(\frac{1}{2} \varepsilon_{\rho \mu \nu \beta} \varepsilon^{\rho \sigma \gamma \lambda} D_{\sigma} B_{\gamma \lambda}^{I}\right)\left(\alpha B_{I}^{\mu \nu}-2 \frac{\delta S}{\delta B_{\mu \nu}^{I}}\right) \\
& +\delta x^{\beta} B_{\beta \mu}^{I}\left[\frac{\delta S}{\delta A_{\mu}^{I}}-2 D_{\nu}\left(\frac{\delta S}{\delta B_{\mu \nu}^{I}}\right)\right]-\frac{1}{2} \alpha \delta x^{\beta}\left(D_{\beta} B_{\mu \nu}^{I}\right) F_{I}^{\mu \nu} .
\end{aligned}
$$


So, by adding (3.11) and (3.12), we obtain

$$
\begin{aligned}
-\frac{1}{2} \alpha \delta x^{\beta} B_{I}^{\mu \nu} D_{\beta} F_{\mu \nu}^{I}-\frac{1}{2} \alpha \delta x^{\beta}\left(D_{\beta} B_{\mu \nu}^{I}\right) F_{I}^{\mu \nu}= & -\frac{1}{2} \alpha \delta x^{\beta} D_{\beta}\left(B_{\mu v}^{I} F_{I}^{\mu v}\right) \\
= & -\frac{1}{2} \partial_{\beta}\left(\alpha \delta x^{\beta} B_{\mu v}^{I} F_{I}^{\mu \nu}\right) \\
& +\frac{1}{2} \alpha B_{\mu \nu}^{I} F_{I}^{\mu v} \partial_{\beta} \delta x^{\beta} .
\end{aligned}
$$

In this manner, by taking into account (3.10)-(3.13) in the transformed action (3.1), we obtain:

$$
\begin{aligned}
& S^{\prime}= S+\int_{R} d^{4} x \partial_{\nu}\left[\alpha \delta x^{\beta}\left(B_{\beta \mu}^{I} B_{I}^{\mu \nu}-B_{I}^{\mu \nu} F_{\beta \mu}^{I}-B_{\beta \mu}^{I} F_{I}^{\mu \nu}\right)\right] \\
&+\int_{R} d^{4} x \partial_{\beta}\left[\alpha \delta x^{\beta}\left(\frac{1}{4} B_{I}^{\mu \nu} B_{\mu \nu}^{I}-\frac{1}{2} B_{\mu \nu}^{I} F_{I}^{\mu \nu}\right)\right] \\
&+\int_{R} d^{4} x\left[\alpha \delta x^{\beta} \frac{\delta S}{\delta A_{\mu}^{I}} F_{\beta \mu}^{I}-\alpha \delta x^{\beta} B_{\beta \mu}^{I} \frac{\delta S}{\delta A_{\mu}^{I}}+\frac{1}{2} \alpha \delta x^{\beta} B_{I}^{\mu \nu} \Psi_{\mu \nu \beta}^{I}-\frac{1}{2} \delta x^{\beta}\left(\varepsilon_{\rho \mu \nu \beta} \varepsilon^{\rho \sigma \gamma \lambda} D_{\sigma} B_{\gamma \lambda}^{I}\right) \frac{\delta S}{\delta B_{\mu \nu}^{I}}\right. \\
&\left.+\delta x^{\beta} B_{\beta \mu}^{I}\left[\frac{\delta S}{\delta A_{\mu}^{I}}-2 D_{\nu}\left(\frac{\delta S}{\delta B_{\mu \nu}^{I}}\right)\right]\right] \\
&=+\int_{R} d^{4} x\left[\partial_{\beta} J^{\beta}+\delta x^{\beta} \frac{\delta S}{\delta A_{\mu}^{I}} F_{\beta \mu}^{I}-\delta x^{\beta} B_{\beta \mu}^{I} \frac{\delta S}{\delta A_{\mu}^{I}}+\frac{1}{2} \alpha \delta x^{\beta} B_{I}^{\mu \nu} \Psi_{\mu \nu \beta}^{I}\right. \\
&\left.-\frac{1}{2} \delta x^{\beta}\left(\varepsilon_{\rho \mu \nu \beta} \varepsilon^{\rho \sigma \gamma \lambda} D_{\sigma} B_{\gamma \lambda}^{I}\right) \frac{\delta S}{\delta B_{\mu \nu}^{I}}+\delta x^{\beta} B_{\beta \mu}^{I}\left[\frac{\delta S}{\delta A_{\mu}^{I}}-2 D_{\nu}\left(\frac{\delta S}{\delta B_{\mu \nu}^{I}}\right)\right]\right] .
\end{aligned}
$$

Hence, because the action is invariant under Poincare transformation, and if equations of motion hold, we identify the conserved Noether four-current given by

$$
\begin{aligned}
J^{\beta} & =\alpha \delta x^{\gamma}\left(B_{\gamma \mu}^{I} B_{I}^{\mu \beta}-B_{I}^{\mu \beta} F_{\gamma \mu}^{I}-B_{\gamma \mu}^{I} F_{I}^{\mu \beta}\right)+\alpha \delta x^{\beta}\left(\frac{1}{4} B_{I}^{\mu \nu} B_{\mu \nu}^{I}-\frac{1}{2} B_{\mu \nu}^{I} F_{I}^{\mu \nu}\right) \\
& =T^{\beta}{ }_{\gamma} \delta x^{\gamma}
\end{aligned}
$$

where

$$
T^{\beta \gamma}=-\alpha\left[B_{I}^{\beta \mu} B^{I \gamma}{ }_{\mu}-B_{I}^{\beta \mu} F^{I \gamma}{ }_{\mu}-F_{I}^{\beta \mu} B^{I \gamma}{ }_{\mu}-\eta^{\beta \gamma}\left(\frac{1}{4} B_{I}^{\mu v} B_{\mu \nu}^{I}-\frac{1}{2} B_{\mu \nu}^{I} F_{I}^{\mu \nu}\right)\right],
$$

which corresponds to the energy-momentum tensor for the theory under study. It is clear that $T^{\beta \gamma}=T^{\gamma \beta}$, and we have not added by hand any terms to symmetrize the tensor, as opposed 
to Belinfante's method. In order to recover the energy momentum tensor found in (3.1), we use the equation of motion (3.4), and the energy-momentum tensor is reduced to

$$
T_{\mu \nu}=-\alpha\left[F_{I \mu}^{\gamma} F_{\nu \gamma}^{I}-\eta_{\mu \nu} \frac{1}{4} F_{\beta \lambda}^{I} F_{I}^{\beta \lambda}\right] .
$$

Thus, $T_{\mu \nu}$ is the same energy-momentum tensor for [YM] theory obtained by means a different approach. Hence, we confirm that the coupling of two topological theories as (3.1) gives a dynamical theory, in others words, the addition of the BB term in (3.1) breaks down the topological structure of the first BF term, and the addition of the BF-like term to the BB term, breaks down the topological structure as well. The coupling, however, allows the existence of physical degrees of freedom [10], and this fact is manifested in the nonvanishing energy-momentum tensor (3.17). We finish this paper with some remarks; in the literature there is a different way to write [YM] theory as a constrained BF-like theory called Mertellini's model [16]. Martellini's model is a deformation of a BF-like topological field theory where it is possible to show that it gives, on shell, the first-order formulation of [YM] theory, in which the Feynman rules have been studied showing that the standard uv-behaviour is recovered, as well as new nonlocal observables related to the phase structure of the theory can be defined. So, we have presented in this paper other alternative to express [YM] theory as a constrained BF-like theory, and we have established the bases to perform the quantum treatment of the action (2.1); thus, we will compare at quantum level the difference among the action analyzed in this work and the action in Martellini's model.

\section{Conclusions and Prospects}

In this paper, the covariant canonical analysis for Yang-Mills theory written as a BF-like action was performed. By defining a dynamical system with its equations of motion plus an action principle, we constructed a closed and gauge invariant symplectic form which contains all the relevant Hamiltonian description of the covariant phase space. Furthermore, the geometric structure obtained for the theory under study allowed us to calculate the gauge transformations and a symmetric and gauge invariant energy-momentum tensor corresponding on shell to the energy-momentum tensor of [YM] theory. On the other hand, we obtained by using Noether's theorem a symmetric and gauge invariant energymomentum tensor confirming on shell the results obtained in former sections. It is important to comment, that it was not necessary to implement Belinfante's approach in order to symmetrize the energy-momentum tensor. In addition, we saw that the action under study being the coupling of topological terms, it is not topological anymore because there exist physical degrees of freedom. Therefore, with the present work we have established the basis to quantize the theory under study in a covariant framework being a good alternative to understand the symmetries of this BF-like action at quantum level, all these ideas are already in progress and will be reported in forthcoming works.

\section{Acknowledgments}

This work was supported by CONACyT México and Sistema Nacional de Investigadores México. 


\section{References}

[1] A. Hanson, T. Regge, and C. Teitelboim, Constrained Hamiltonian Systems, Accademia Nazionale dei, Roma, 1978.

[2] M. Blagojević, Gravitation and Gauge Symmetries, Series in High Energy Physics, Cosmology and Gravitation, IOP Publishing, Bristol, UK, 2002.

[3] C. Crncovic and E. Witten, Three Hundred Years ofGravitation, edited by S. W. Hawking, Cambridge University Press, Cambridge, Mass, USA, 1987.

[4] C. Crncovic, "Symplectic geometry and super-algebra in geometrical theories," Nuclear Physics B, vol. 288, pp. 419-430, 1987.

[5] R. Cartas-Fuentevilla and A. Escalante, "Topological terms and the global symplectic geometry of the phase space in string theory," in Trends in Mathematical Physics Research, pp. 95-111, Nova Science Publishers, Hauppauge, NY, USA, 2004.

[6] M. Montesinos and G. F. Torres del Castillo, "Symplectic quantization, inequivalent quantum theories, and Heisenberg's principle of uncertainty," Physical Review A, vol. 70, no. 3, Article ID 032104, 8 pages, 2004.

[7] A. Escalante, "The Chern-Simons state for topological invariants," Physics Letters B, vol. 676, no. 1-3, pp. 105-111, 2009.

[8] A. Escalante and J. Angel López-Osio, "Hamiltonian analysis for topological and Yang-Mills theoriesas a constrained BF-like theory," International Journal of Pure and Applied Mathematics, vol. 75, pp. 339-352, 2012.

[9] M. Mondragón and M. Montesinos, "Covariant canonical formalism for four-dimensional BF theory," Journal of Mathematical Physics, vol. 47, no. 2, Article ID 022301, 15 pages, 2006.

[10] A. Escalante and J. Berra-Montiel, "A pure Dirac's method for Yang-Mills theory expressedas a conBF-like theory," submitted to Physics Letters B.

[11] W. Greiner and J. Reinhardt, Field Quantization, Springer, Berlin, Germany, 1996, Translated from the German, with a foreword by D. A. Bromley.

[12] M. Montesinos and E. Flores, "Symmetric energy-momentum tensor in Maxwell, Yang-Mills, and Proca theories obtained using only Noether's theorem," Revista Mexicana de Física, vol. 52, no. 1, pp. 29-36, 2006.

[13] C. Rovelli, Quantum Gravity, Cambridge Monographs on Mathematical Physics, Cambridge University Press, Cambridge, Mass, USA, 2004, With a foreword by James Bjorken.

[14] T. Thiemann, Modern Canonical Quantum General Relativity, Cambridge Monographs on Mathematical Physics, Cambridge University Press, Cambridge, Mass, USA, 2007, With a foreword by Chris Isham.

[15] A. Ashtekar, Lectures on Nonperturbative Canonical Gravity, vol. 6 of Advanced Series in Astrophysics and Cosmology, World Scientific, River Edge, NJ, USA, 1991, In collaboration with R. S. Tate.

[16] M. Martellini and M. Zeni, "Feynman rules and $\beta$-function for the BF Yang-Mills theory," Physics Letters B, vol. 401, no. 1-2, pp. 62-68, 1997. 


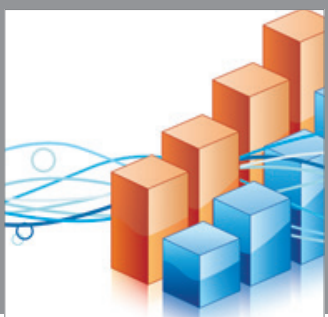

Advances in

Operations Research

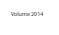

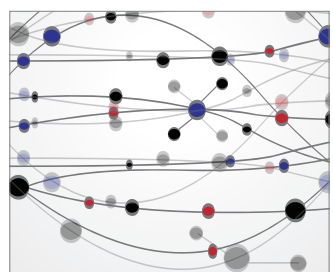

\section{The Scientific} World Journal
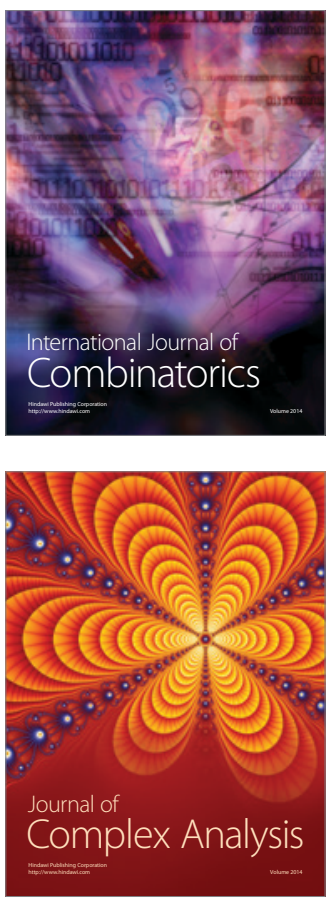

International Journal of

Mathematics and

Mathematical

Sciences
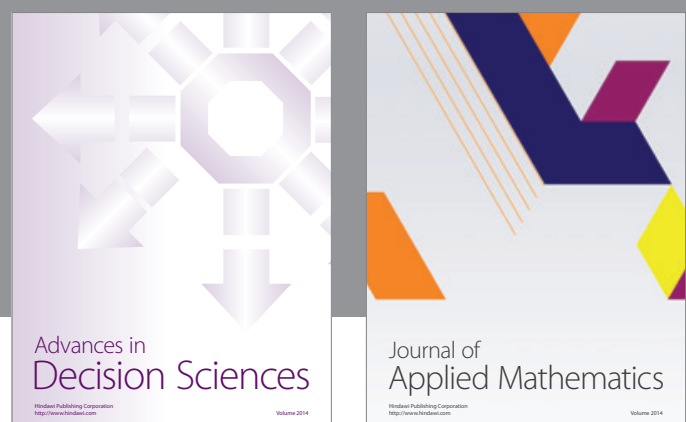

Journal of

Applied Mathematics
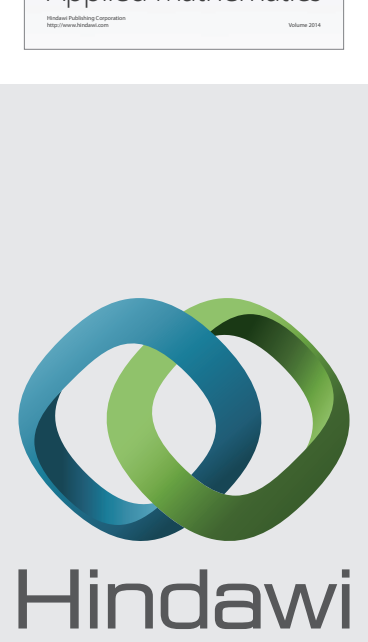

Submit your manuscripts at http://www.hindawi.com
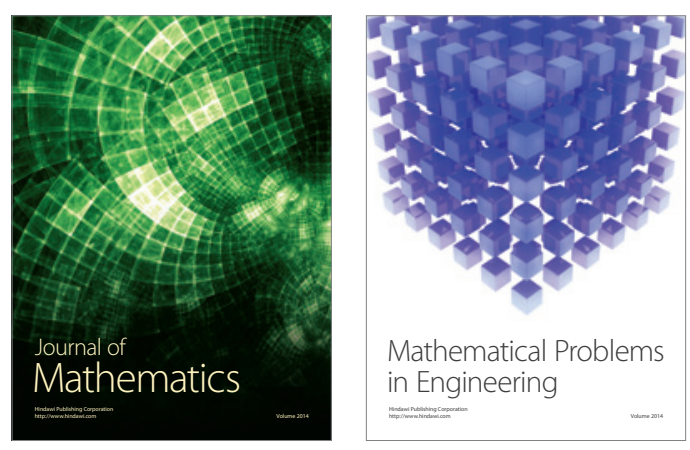

Mathematical Problems in Engineering
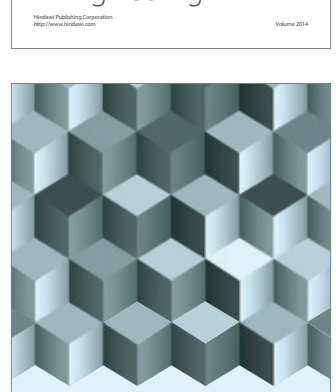

Journal of

Function Spaces
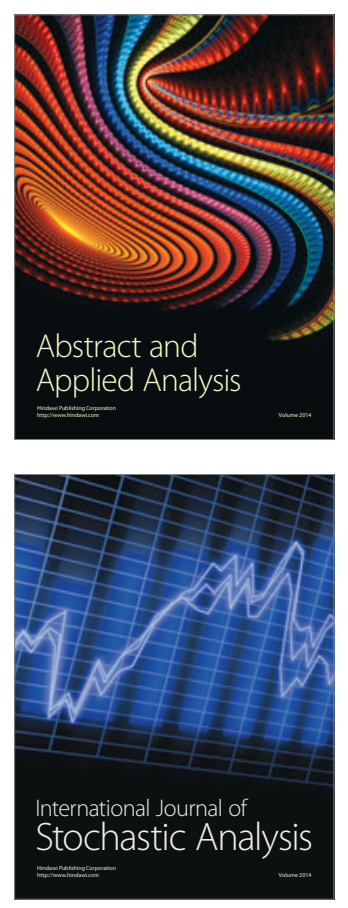

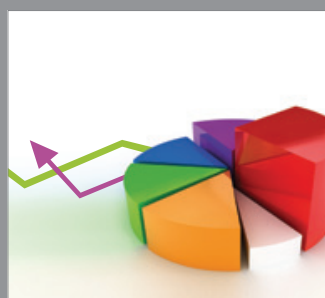

ournal of

Probability and Statistics

Promensencen
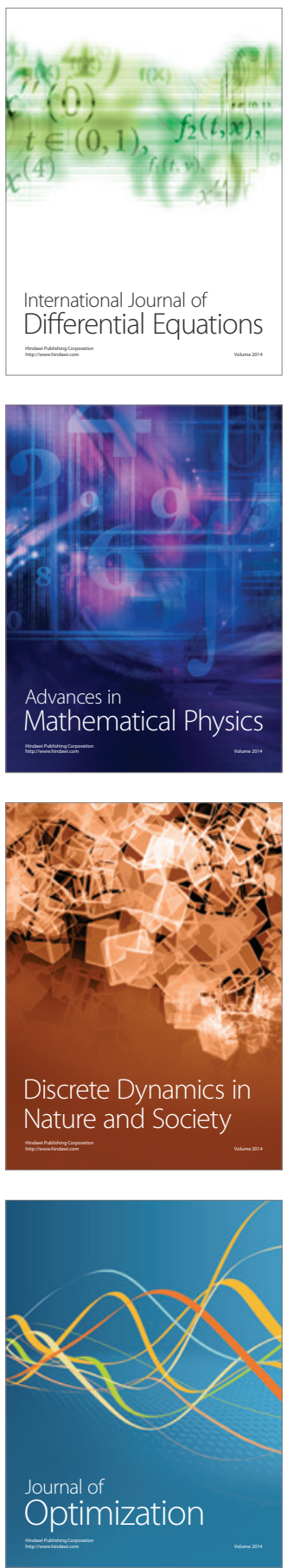